\title{
Formation of Monolayer Polystyrene Array Template for Gold Nanomesh Structure
}

\author{
Nur Liyana Razali 1, 2, a), Marlia Morsin", 2, b)*, Muhamad Mat Salleh ${ }^{3, c),}$ \\ Nurfarina Zainal 1, 2, d), Marriatyi Morsin ${ }^{3, e)}$
}

\author{
${ }^{1}$ Institute of Microelectronics \& Nanotechnology - Shamsuddin Research Centre (MiNT-SRC), \\ Universiti Tun Hussien Onn Malaysia,86400, Parit Raja, Batu Pahat, Johor, Malaysia. \\ ${ }^{2}$ Faculty of Electrical and Electronic Engineering, Universiti Tun Hussien Onn Malaysia, \\ 86400, Parit Raja, Batu Pahat, Johor, Malaysia \\ ${ }^{3}$ Institute of Microengineering and Nanoelectronics (IMEN), Universiti Kebangsaan Malaysia, \\ 43600 UKM Bangi, Selangor, Malaysia \\ ${ }^{4}$ Politeknik Sultan Salahuddin Abdul Aziz Shah, Persiaran Usahawan, Seksyen U1, 40150 Shah Alam, Selangor.
}

Received 31 December 2017; accepted 6 June 2018, available online 6 August 2018

\begin{abstract}
In this study, the preparation of monolayer array template was performed using $0.2 \mu$ m polystyrene (PS) nanospheres monodisperse micro particle deposited onto Indium Tin Oxide (ITO) coated glass substrate. The template was used to arrange the gold $(\mathrm{Au})$ in nanomesh structure thus, enhance its homogeneity for having better sensitivity and repeatability in the plasmonic sensor application. PS nanosphere template was prepared by diluting $0.2 \mu \mathrm{m}$ PS liquid with ethanol in the ratio of $1: 3$ and stirred with $400 \mathrm{rpm}$ for 1 hours at $40{ }^{\circ} \mathrm{C}$. Next, the PS solution was spin coated on ITO substrate at different speed variation starting from $200 \mathrm{rpm}$ to $800 \mathrm{rpm}$ in 3 minutes at room temperature. The template structure was then inspected with Field Emission-Scanning Electron Microscopy (FESEM). It was found that, the PS nanosphere template with $600 \mathrm{rpm}$ speed shows the most orderly arrangement of PS array. Subsequently, the PS array template was used as a mask to deposit gold (Au) and performed via direct current (DC) sputtering method. Finally, the Au nanomesh structure was obtained using the lift - off process of PS nanosphere template.
\end{abstract}

Keywords:

Localized Surface Plasmon Resonance, Array template, Polystyrene (PS) Nanosphere, Gold Nanoparticles,

\section{Introduction}

There are various type of metal nanoparticles (NPs), such as gold $(\mathrm{Au})$, silver $(\mathrm{Ag})$ and copper $(\mathrm{Cu})$ which had demonstrated exceptional radiative, nonradiative and optical properties, reasonable for an extensive variety of utilization including disease detection, diagnosis and therapy, drug delivery, biomedical imaging, biosensing [1-2] and many more. These metal NPs are popular and widely used because of their chemical properties, surface plasmon behavior and nanometer (nm) in size. Apparently, gold nanoparticles is the most commonly used due to its simple and easy preparation and synthesizing method.

Localized surface plasmon resonance (LSPR) is a resonance phenomenon of free electron waves in a metal nanostructure [3]. Gold nanostructure is one of the metal nanostructures that have gained much attention in the field of electronics, non - linear optic applications. Thus far, many researches of gold nanostructures have been done in form of synthesis and its functionality [4-6]. However, assembly or alignment of gold nanostructures and inter - particle separation are still requires further investigations. The need of gold particles arrangement is a crucial element in order to enhance its sensitivity and repeatability. In the case of optical sensor applications, aggregation of gold nanoparticles can affected particles alignment hence produce insensitive optical device with poor sense performance. To date, many methods were used to compose particle arrangement such as lithography (printed pattern) [7], implementing various surfactant [8] and also block of co - polymers [9]. Yet, several of these methods are complex and require a special intensive care and costly.

In this study, assembly or alignment of gold nanostructures and inter - particle separation was performed using a lift - off technique. This technique was chosen due to simpler method and inexpensive as compared to other methods mention above. Monolayer array template was produced using polystyrene (PS) nanospheres monodisperse micro particle which 
deposited onto ITO coated glass substrate. The template was used to arrange the gold ( $\mathrm{Au}$ ) in nanomesh structure with the purpose to enhance its homogeneity for LSPR sensor application or also known as plasmonic sensor.

\section{Experimental work}

The gold $(\mathrm{Au})$ nanomesh structure was fabricated using three main procedures such as preparation of polystyrene (PS) template, sputtering of gold on the ITO coated glass substrate and removal of polystyrene template from the ITO coated glass substrate. These processes are called as lift - off technique and employed the usage of deionized (DI) water with resistivity $18.2 \mathrm{M} \Omega \mathrm{cm}$.

First, the polystyrene (PS) template was prepared by diluting $0.2 \mu \mathrm{m}$ PS particle liquid with ethanol in a ratio of $1: 3$. The PS mixture solution was then stirred for 1 hour at $40{ }^{\circ} \mathrm{C}$ with $400 \mathrm{rpm}$ and spin coated onto the substrate. The $0.2 \mu \mathrm{m}$ polystyrene monodisperse micro particle was purchased from Sigma Aldrich. The speed of stirring times were varied in the range of 200 until 800 rpm for 3 minutes.
Next, gold is transferred onto the template using direct current (DC) sputtering system at room temperature for 4 minutes. In this research, PolaronHigh Resolution sputter coater was used with gold $\mathrm{(Au}$ ) solid source. The gold sputtering deposition process was performed in rotation a form with setting parameter of 2.5 $\mathrm{kV}$ voltage and $25 \mathrm{~mA}$ current. The gold solid source was purchased from $\mathrm{Hi}$ - Tech Instruments. Finally, the polystyrene (PS) nanosphere template was removed from the substrate using acetone. The template removal procedure was through in three different phases for example 15, 30 and 60 seconds under sonication processes. Following of nanosphere PS template removal, surface morphology of the gold nanomesh structures were inspected and observed using FESEM Zeiss Supra 55VP. Figure 1 show the schematic diagram of lift-off processes that used to fabricate the gold nanomesh structure using polystyrene nanosphere template.
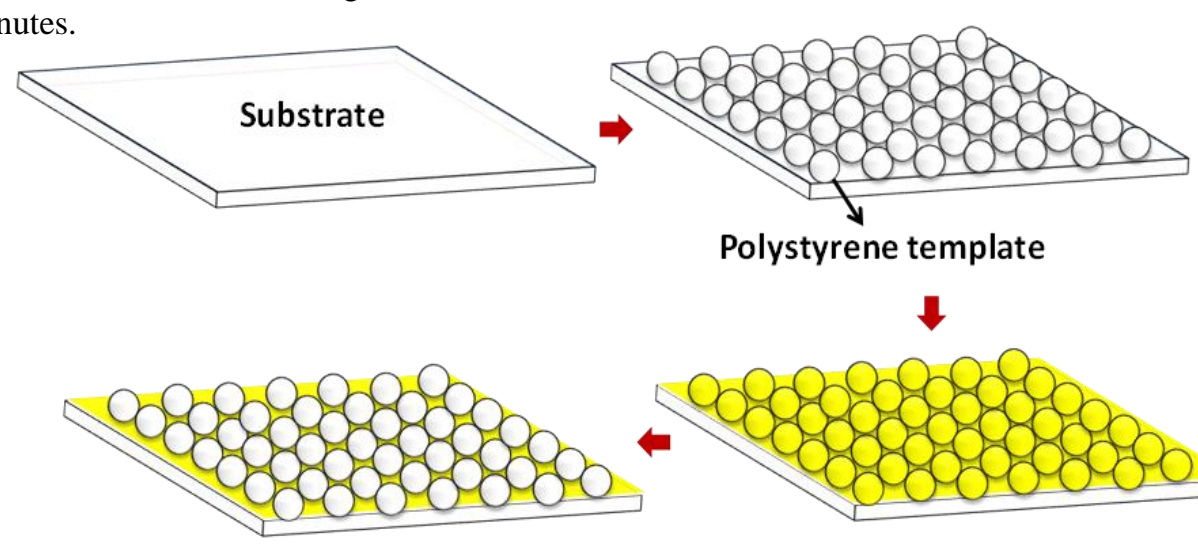

After template removal

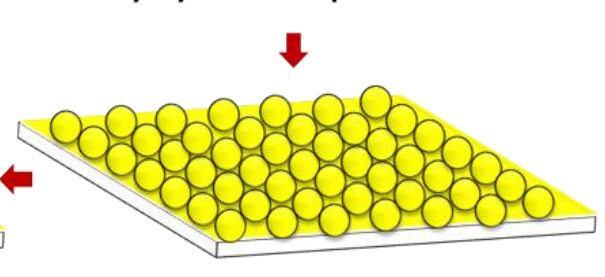

After gold sputtered

Figure 1: Schematic diagram of gold (Au) nanomesh structure using polystyrene (PS) nanosphere template in a lift - off technique.

\section{Results and Discussions}

As discussed above, there were three steps involving in the lift - off processes. Begin with the preparation of polystyrene (PS) template by construct a PS monolayer template on the substrate surface so that gold nanomesh structure can be arranged accordingly. Stirred mixture of PS and ethanol solutions were aimed to break the aggregation after the dilution procedure. It has reported [10-12] different stirring times can affected the PS arrangement. Thus, in this research 4 different stirring phases were applied for instance 200, 400, 600 and 800 rpm. Each of samples were then examine using FESEM as shown in Fig. 2.

For $200 \mathrm{rpm}$ speed, it can be observed that the PS layer seems inhomogeneity with stackable nanosphere. Once the stirrer speed was increased, the homogeneity of the PS nanosphere has been improved as in Fig. 2 (B) andC). However, when the speed was extended to $800 \mathrm{rpm}$, the PS nanosphere looks more scattered as in Fig. 2 (D). In comparison, the most orderly PS nanosphere template arrangement with $600 \mathrm{rpm}$ speed. Thus, sample with 600 rpm stirring speed was chosen to be coated with gold layer.

For second step, sputtered gold layer was deposited onto the PS nanosphere template with $600 \mathrm{rpm}$ stirring phases. In this case, two different gold sputtering deposition approaches were carried out with samples in the rotated and fixed platform. The sputtering rate was around $15 \mathrm{~nm} / \mathrm{mins}$ which resulting thickness of gold ca. $60 \mathrm{~nm}$. In reality the yellow color of gold was observed on the substrate after sputtering process completed. Then, the samples were checked again using FESEM as depicted in Fig. 3. 
(A)

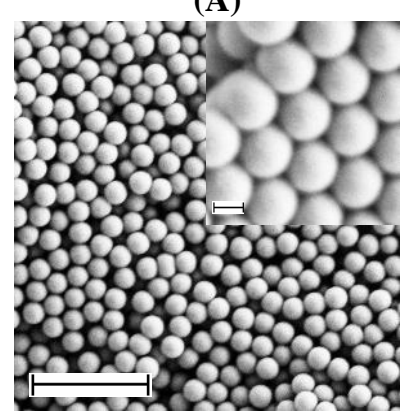

(C)

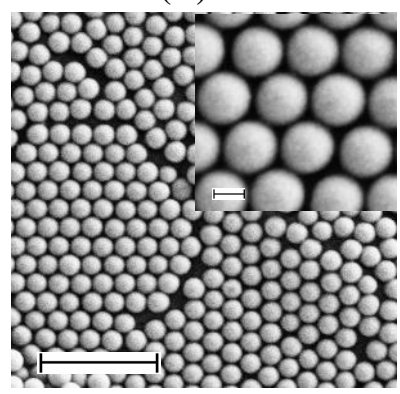

(B)

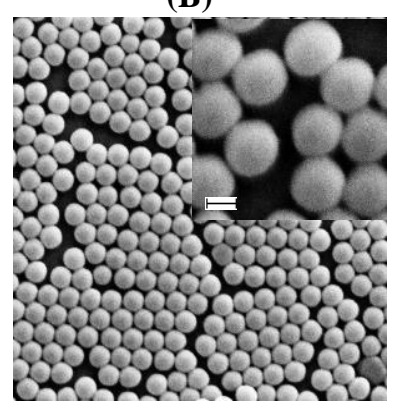

(D)

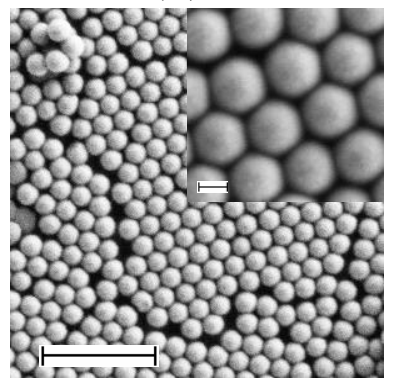

Fig. 2. FESEM images of Polystyrene (PS) nanosphere template with different coating speed (A) $200 \mathrm{rpm}$, (B) $400 \mathrm{rpm}$, (C) $600 \mathrm{rpm}$, and (D) $800 \mathrm{rpm}$. Scale for image: $1 \mu \mathrm{m}$ and inset: $100 \mathrm{~nm}$.

The images show samples after gold coated on the PS nanosphere template using DC sputtering method. Fig. 3(A) shows the gold was uniformly coated on the PS nanosphere surface with darker image by using rotated platform. On the other hand, fixed sputtering platform produced inhomogeneous gold distribution as shown in Fig. 3 (B). It can be clearly seen in the Fig. 3 (B) inset that surface morphology of fixed sputtered samples was nonuniform or uneven. It is believed that the inhomogeneous distribution of gold layer can be caused by many factors such as low current and voltage applied, the fixed platform is not suitable and size of template are not well suited. Bear in mind, the size of template must be well selected to ensure all the surface samples are covered up with gold layer until the substrate surface. In this research, the surface density of the polystyrene particle is ca. $575 / \mathrm{um}^{2}$. The measured size of polystyrene particles is ca. $0.2 \mu \mathrm{m}$; which is same with the size given by manufacturer. Based on the density and size measurement results, it can be concluded that the distribution of gold on PS nanosphere template is not really in homogeneous condition which covered up to the substrate layer. Besides, the gold layer was not all covered in all spaces between PS nanosphere but rather coated just around the PS surface. Even this so, the experiments were conducted until the removal procedure.

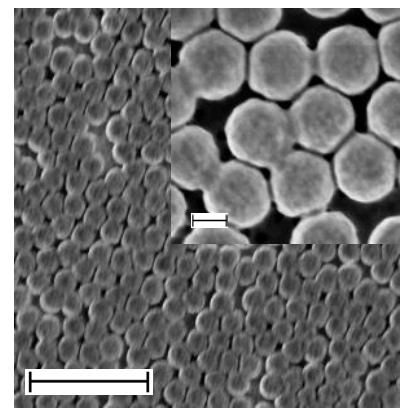

(A)

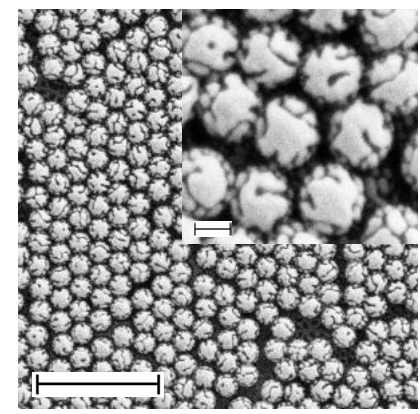

(B)
Fig. 3. After gold sputtering onto the PS template using (A) Rotation platform (B) Fixed platform. Scale for image: $1 \mu \mathrm{m}$ and inset: $100 \mathrm{~nm}$.

Lastly, the removal of polystyrene template from the substrate was proceeded or also known as lift-off process. The PS nanosphere template was removed using acetone for 3 different removal times for example 15 seconds, 30 seconds and 60 seconds. The results are shown in Fig. 4.

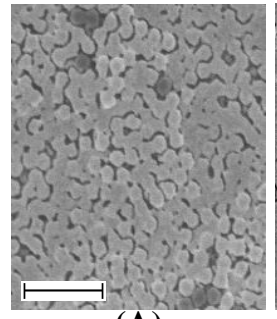

(A)

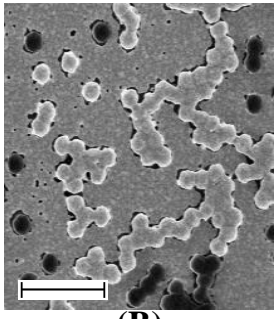

(B)

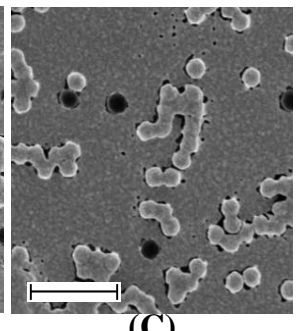

(C)
Fig. 4. After PS template removal using acetone for three different times (A) 15 seconds (B) 30 seconds and (C) 60 seconds. Scale for image: $1 \mu \mathrm{m}$

The PS template was removed from the substrate surface. However, the formation of gold $(\mathrm{Au})$ nanomesh structure using polystyrene (PS) template was not fully transferred on the surface due to strong attraction between PS nanosphere and gold. The attraction is related with the electrostatic charging between these two types or particles. As a result, the sputtered gold was not placed in the space provided between PS nanosphere intersection areas but coated only on its top surface as mentioned before.

\section{Conclusion}

The formation of gold $(\mathrm{Au})$ nanomesh structure using polystyrene (PS) template was not very suitable to be used in formed the gold nanomesh structure. This is because of strong attraction between PS nanosphere and gold can cause the gold not deposited in the space provided between PS particles (intersection) but only coated on its surface. As a suggestion, the PS nanoshere can be used to make as template and gold sputtering method can be replaced with synthesizing gold using wet chemical method. 


\section{Acknowledgements}

This work was supported by Universiti Tun Hussein Onn Malaysia under UTHM Contract Research Grant (U565UTHM) and IMEN, Universiti Kebangsaan Malaysia for the laboratory facilities.

\section{References}

[1] Y. Zhang, J. Xiao, Y. Sun, L. Wang, X. Dong, J. Ren, W. He, F. Xiao, "Flexible nanohybrid microelectrode based on carbon fiber wrapped by gold nanoparticles decorated nitrogen doped carbon nanotube arrays: In situ electrochemical detection in live cancer cells," Biosens.

Bioelectron., vol. 100, September 2017, pp. 453461, 2018.

[2] B. F. G. Vadim V. Sumbayev, Inna M. Yasinska, "Biomedical Applications of Gold Nanoparticles," Recent Adv. Circuits, Commun. Signal Process. Biomed., vol. 1, no. 2, pp. 10-25, 2012.

[3] E. Hutter and J. Fendler, "Exploitation of Localized Surface Plasmon Resonance", Advanced Materials, vol. 16, no. 19, pp. 1685-1706, 2004.

[4] T. Premkumar, K. Lee and K. Geckeler, "Shapetailoring and catalytic function of anisotropic gold nanostructures", Nanoscale Research Letters, vol. 6, no. 1, p. 547, 2011.

[5] M. Stewart, C. Anderton, L. Thompson, J. Maria, S. Gray, J. Rogers and R. Nuzzo, "Nanostructured Plasmonic Sensors", Chemical Reviews, vol. 108, no. 2, pp. 494-521, 2008.

[6] M. Vidotti, R. Carvalhal, R. Mendes, D. Ferreira and L. Kubota, "Biosensors based on gold nanostructures", Journal of the Brazilian Chemical Society, vol. 22, no. 1, pp. 3-20, 2011.

[7] P. Maury, M. Escalante, D. Reinhoudt and J. Huskens, "Directed Assembly of Nanoparticles onto Polymer-Imprinted or Chemically Patterned Templates Fabricated by Nanoimprint Lithography", Advanced Materials, vol. 17, no. 22, pp. 2718-2723, 2005.

[8] X. Zhou, J. Li, C. Liu and L. Jiang, "Preparation, stability and two-dimensional ordered arrangement of gold nanoparticles capped by surfactants with different chain lengths", Science in China Series B: Chemistry, vol. 45, no. 4, pp. 358-364, 2002.

[9] H. Yabu, T. Jinno, K. Koike, T. Higuchi and M. Shimomura, "Nanoparticle arrangements in block copolymer particles with microphase-separated structures", Journal of Polymer Science Part B: Polymer Physics, vol. 49, no. 24, pp. 1717-1722, 2011.

[10] A. Kotera, K. Furusawa and K. Kudō, "Colloid chemical studies of polystyrene latices polymerized without any surface-active agents", Kolloid-Zeitschrift \& Zeitschrift für Polymere, vol. 240, no. 1-2, pp. 837-842, 1970.

[11] T. Ghoshal, A. Chaudhari, C. Cummins, M. Shaw, J. Holmes and M. Morris, "Morphological evolution of lamellar forming polystyrene-blockpoly(4-vinylpyridine) copolymers under solvent annealing", Soft Matter, vol. 12, no. 24, pp. 54295437, 2016.

[12] A. Rose, "The fabrication of polystyrene mouldings for use as a reduced density hydrogenous moderator", Nuclear Instruments and Methods, vol. 77, no. 1, pp. 167-169, 1970. 